\title{
Carl Max Rebel und Stefan George
}

\section{Eine Skizze}

„E]r hatte neue Stiefel an, so dass ihm die Füsse weh taten. Als ich mich lachend erbot, inn die fünf Treppen hinaufzutragen, meinte er lächelnd: ,Das wäre so etwas für die Philister!'” Die beiden Protagonisten dieser wohl 1896 spielenden Szene sind der Maler Carl Max Rebel und der Lyriker Stefan George, die sich im Berliner Café Klose, Leipzigerstraße 19, kennengelernt haben und aufgebrochen waren zu Rebels gut dreißig Fußminuten entfernt liegendem Atelier in der Elsasserstraße 21 (der heutigen Torstraße), um Rebels frühe Werke anzuschauen. Rebel erinnert: "Er sah bei mir: die Abendlandschaft, den Ritter Zendelwald, das Abdante, die Johannisnacht, den Morgen und vieles andere [...]. - Dann gingen wir zum Kaffee Klose [...] zurück (trotz der Stiefel), wo wir Monate hindurch fast jeden Abend zusammentrafen."1

Carl Max Rebel, am 9. Mai 1874 in Berlin geboren, hatte einige Zeit an der Königlichen Akademie Malerei studiert und war wohl über seinen ehemaligen Studienkollegen Melchior Lechter ins Berliner Umfeld Georges gekommen. Das Malerehepaar Sabine und Reinhold Lepsius, der Archäologe Botho Graef und Karl Wolfskehl etwa lernen Rebel so schon vor 1897 kennen. Im Februar 1900 ist es dann auch ein Bekannter aus diesem Umfeld, der junge Schriftsteller Ernst Hardt (und seit 1898 Mitarbeiter der Dresdner Zeitung), der Rebel eine erste Ausstellung von 20 Bildern im Kunstsalon Ernst Arnold, Dresden, vermittelt. Und noch bevor Georges ehemaliger Darmstädter Schulkollege Ge-

1) Hier und zuvor: Carl Max Rebel: Lebenserinnerungen. Auszug aus dem Manuskript. 7-seitiges Typoskript v. 24.2.1949 (Beilage zu Walther Greischel an Robert Boehringer v. 28.02.1968; StGA: Boehringer III, 10834a), hier S. $1 f$.

2) Georg Fuchs: Carl Max Rebel - Berlin. In: Deutsche Kunst und Dekoration 9 (1901/02), S. 251-263, hier S. 251 (https://digi.ub.uni-heidelberg. de/diglit/dkd1901_1902/0263). Vgl. ebd. auch die späteren, ebenfalls reich bebilderten Artikel von Wolfgang von Oettingen: Carl Max Rebel. In: DKuD 17 (1905/06), S. 197-203 (https://digi.ub.uni-heidelberg.de/ diglit/dkd1905_1906/0203), Karl Widmer: Farbe und Raumstimmung. In: DKuD 17 (1905/06), S. 204-209 (https://digi.ub.uni-heidelberg.de/ diglit/dkd1905_1906/0210) u. Friedrich Spiro: Carl Max Rebel - Rom. In: DKuD 26 (1910), S. 382-387 (https://digi.ub.uni-heidelberg.de/diglit/ dkd1910/0398)

3) Carl Max Rebel an Stefan George, 14.8.1901 (StGA: George III, 10271).

4) Vgl. zur Kunstrezeption Georges: Michael Thimann: Bildende Kunst. In: Stefan George und sein Kreis. Ein Handbuch. Hg. v. A. Aurnhammer u.a. Berlin u. Boston 2012, Bd. 2, S. 551-584.

5) So umriss Wolfgang von Oettingen 1906 in Deutsche Kunst und Dekoration (wie Anm. 2), S. 200, Rebels Movens. org Fuchs, seit 1891 als freier Autor für die Wahrnehmung der darstellenden und bildenden Künste engagiert, den Maler 1902 dann in einem vielfach bebilderten Artikel in Deutsche Kunst und Dekoration einem breiteren Publikum vorstellt, ${ }^{2}$ hat Rebel George in seinem Elternhaus in Bingen besucht und wird von diesem mit ausführlichen Informationen für eine Reise in die Niederlande, mit Tipps zu Reisewegen und Gemälden versorgt. Am 14.8.1891 dankt Rebel aus Schreveningen: „Lieber Herr George! Der Saul [von Rembrandt] ist malerisch die grösste Offenbarung $\ulcorner$ für mich $\urcorner$ gewesen, haben Sie überhaupt Dank für alles. [...] Das Moritzhaus ist gar keine Gallerie, sondern ein ganzer Himmel voller Herrlichkeiten, van Delft, Rembrandt, Ter Borch, Wouwerman, Hans Holbein (wundervolles Portrait mit Falken). Ich war volle 4 Stunden da u. gehe gleich nochmal hinein, ich bin überhaupt ganz toll von diesem wundervollen Land, ich wünschte Sie wären nur auch noch hier!" 3

Trotz der zahlreichen verbindenden Elemente - der fundamentalen Entscheidung zu einem Leben als freischaffende Künstler etwa, dem Interesse an Kunsterneuerung oder demjenigen an bildhaftem Sehen, das auch Georges frühes Werk prägt ${ }^{4}$, gibt es bald darauf allerdings schon keine Belege mehr für direkten Kontakt zwischen George und Rebel. Es mag schlicht daran gelegen haben, dass sich Rebels Lebensschwerpunkt in den Jahren nach seiner Hochzeit im April 1903 (bei seiner Frau Isy handelte es sich wohl um die Schwester seines Malerkollegen Curt Stoeving, auch er ein GeorgeBekannter und -Portraitist) bis 1909 für zwei Mal zwei Jahre nach Rom verschiebt, wo er, kopierend, die italienische Kunst studiert. „Rebels zur Mystik geneigte[r], von Literatur erregte[r], von allem Elegisch-Wundersamen leicht ergriffene[r] Sinn" aber, ${ }^{5}$ der bis dahin vor allem neuromantische Märchenund Legenden-Szenen, jugenstilhafte Allegorien und Landschaftsbilder gemalt, sich in Italien dann auch gelinde realistischeren Sujets angenähert, über die Jahre hinweg aber immer auch im Portrait gearbeitet hatte, entdeckt nach 1909 dann, zurück in Berlin, George als Sujet. Oder vielmehr George 
als seinerzeit ebenso modische wie marktfähige Symbolfigur eines heroischen Dichtertums. 1912 formuliert der Heidelberger Germanist Friedrich Gundolf es gegenüber George einmal so (Anlass ist die Frage nach der Verwendung einer Photographie): „dein kopf [ist] längst nicht mehr deine Privatsache [...], sondern ein Werk zur Reinigung der Begriffe vom Menschen ". ${ }^{6}$ Photographien spielen dabei nicht nur im semi-privaten Umfeld der George-Anhänger eine ikonenhafte Rolle, sondern die Lichtbilder sind nach 1900 auch Mittel von Georges öffentlicher Selbstvermarktungs- und Medienstrategie. Vor allem die Portraits des ehern ernsten, abweisend statischen George als streng-undurchschaubarem Widerständler gegen den Zeitgeist, aufgenommen von den Binger Photographen Jacob und Theodor Hilsdorf, brennen sich von da ab in das Bildgedächnis des frühen Zwanzigsten Jahr-

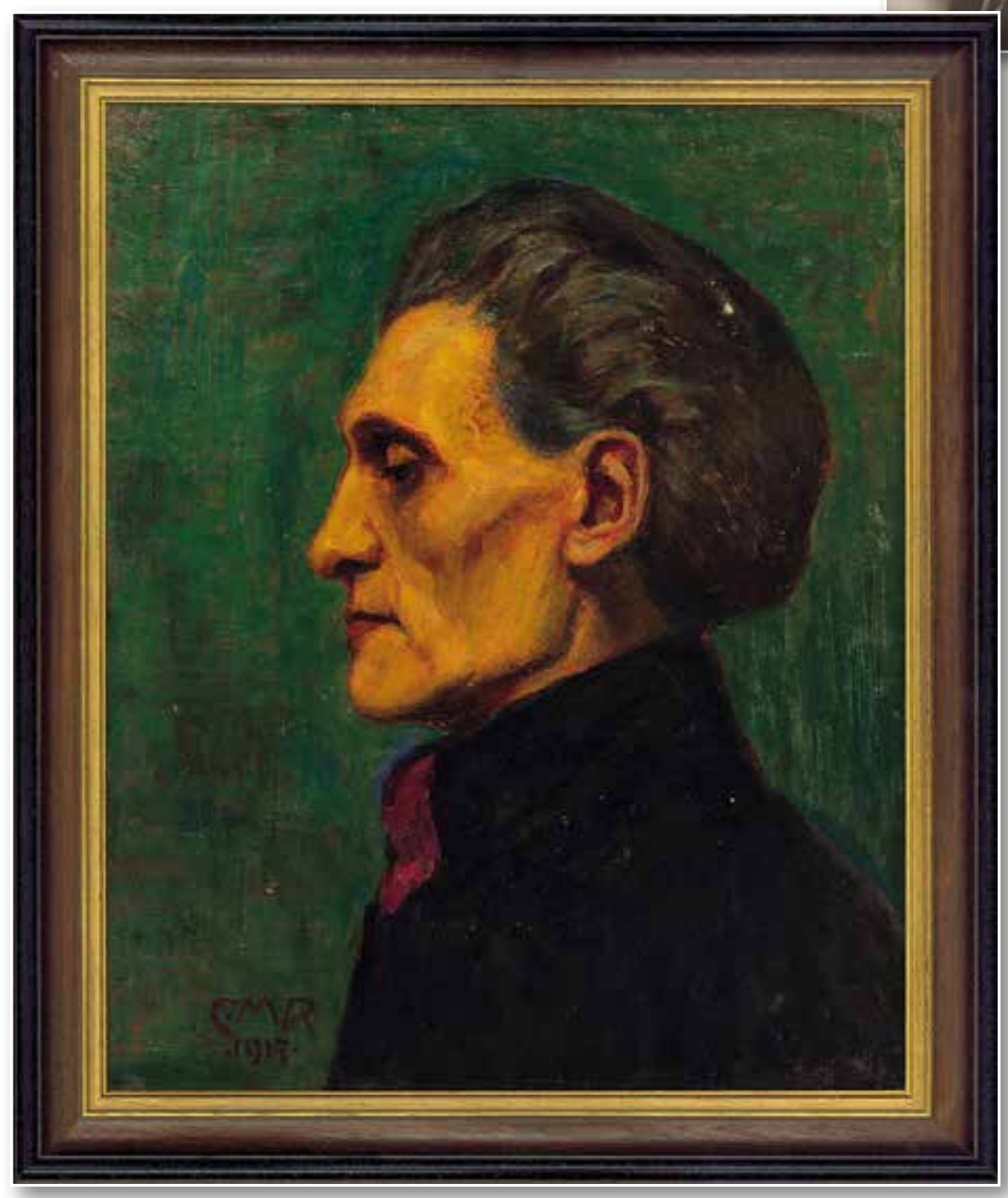

genössisch-moderner Arbeitsweise gearbeitet. Denn keines von Rebels George-Bildern entsteht nach dem lebenden Vorbild. Vorlage für die allermeisten Bilder sind eben HilsdorfPhotos, die Rebel mitunter etwas frei interpretiert oder Charakteristika einzelner Aufnahmen kombiniert. So läßt sich als Vorlage für das Gemälde, das im November 2019 für die Sammlungen des Stefan George Archivs aus privater Hand erworben werden konnte (vgl. die Abbildung links), von Rebel auf 1917 datiert ist und in seiner beinahe spätexpressionistischen Farbgebung überrascht, recht wahrscheinlich eine der berühmtesten Hilsdorf-Aufnahmen von George identifizieren, die 1910 entstanden ist (vgl. die Abbildung oben). Dabei folgen die Kopfhaltung oder der Winkel der niedergeschlagenen Augen des Dargestellten eher Varianten der Aufnahme aus demselben Shooting Hilsdorfs; für die auffällig gestaltete Schläfenader

hunderts ein. Carl Max Rebels Portraits von George nehmen Teil an dieser Kultivierung des Dichters als Idol der Modernekritik und sind dabei doch in zeit- könnte Rebel zudem Anleihen bei weiteren bekannten Photographien genommen haben. 
Stefan George selbst hat wohl nie eines von Rebels George-Portraits gesehen. Noch 1931 fragt der inm selbst bereits fernstehende Melchior Lechter einmal an, ob George nicht ein neu entstandenes "SCHÖNES, GROSSEMPFUNDENES UND GESTALTETES BILDNIS" seiner selbst in Rebels Atelier ansehen wolle.? George reagierte wohl gar nicht erst auf die Anfrage... Als Rebel aber dann knapp vier Jahre nach Georges Tod 1937 neben 24 Landschaftsbildern, Blumenstilleben und Pastellen bei einer Einzelausstellung in der Galerie Gurlitt in Berlin auch 10 seiner George-Portraits ausstellt, wird das nicht nur in der Presse besprochen, sondern auch von den George-Vertrauten und -erben wahrgenommen: Frank Mehnert, Georges später Lebensgefährte, selbst Plastiker und im NS-Staat dann mit Bismarckund Hitler-Büsten, dem Entwurf zu einem SADenkmal oder dem Magdeburger Pionierstandbild mit den Zügen Claus Graf Stauffenbergs öffentlich dienlich, hinterlässt Bemerkungen auf einem Faltblatt zur Ausstellung. Nicht nur zieht er die Datierungen in Zweifel, wenn er auf das Alter der von Rebel benutzten Bildvorlagen verweist: „Bildnis 1909: Schwindel. nach einem Hilsdfbild v. 1910!" oder "Altersbild 1933: anderes Hilsdfbild 1928". Er hält auch mit seiner ästhetischen Einschätzung nicht zurück, nennt Bilder "entsetzlich.." oder "grausig" und scheint dem Maler über eine notierte feindliche Sotise nachträglich jede Berechtigung für dessen ,Arbeit am Bild Georges' entziehen zu wollen: „bei spaziergängen im Westen Berlins in der gegend der Achenbachstr. [dort hatte Rebel nach 1909 lang sein Atelier, M.B.] sei $\lceil$ habe es $\urcorner$ vorkommen können · dass d.M. [,der Meister', d.i. George] seinen begleiter plötzlich in eine nebenstrasse zog mit den worten: schnell · da kommt so eine von Lechters atelierwanzen." 8

Die öffentliche Wahrnehmung von Rebels GurlittAusstellung 1937 dagegen ist durchaus positiv

7) Melchior Lechter und Stefan George: Briefe. Kritische Ausgabe. Hrsg v. G. Heintz. Stuttgart 1991, S. 330 (Brief v. 19.2.1931).

8) Vgl. Notizen Frank Mehnerts auf dem Faltblatt zur Ausstellung Rebel, Galerie Gurlitt 1937 (StGA-Kunst-0217).

9) Richard Groeper: Carl Max Rebel und seine Stefan-George-Bilder. In: Deutscher Kulturwart 4 (1937), Juli-Heft, S. 391-394, hier S. 392. Vgl. auch Alfred Jürgens: Carl Max Rebel - ein Malerleben für den Dichter Stefan George. In: Ostdeutsche Monatshefte 18.8 (1937), o.S., o. Johannes Günther: Zu den Gemälden des Berliner Malers Carl Max Rebel. In: 8 Uhr Abendblatt v. 13. April 1937.

10) Fritz Nemitz: Stefan George im Bildnis. Carl Max Rebel bei Gurlitt. In: BZ v. 14.11.1937

11) "[B]is zum Jahre 1909 ha[tt]e ich etwa 40 Kollektivausstellungen zu verzeichnen .... In den folgenden Jahren beschickte ich auch die grosse Berliner und die ,Juryfreie' bis 1927. Dann nach zehnjähriger Pause die Atelierausstellung 1937 und im selben Jahr vom 7. bis 30. November die abschliessende Collektiv-Ausstellung im Salon Gurlitt", Rebel: Lebenserinnerungen (wie Anm. 1), S. 5.
- und beinahe völlig auf die Portraits des, großen Mannes', das Lob der bildlichen Symbolisierung von Georges Lebens- als vermeintlicher Leidensgeschichte für sein Werk und Volk, die nicht selten völkisch durchwirkte Heroisierung des deutschen Dichterdenkers konzentriert (d.h. die ebenfalls ausgestellten und zahlenmäßig überwiegenden Landschaftsbilder oder Blumenstilleben werden in den Artikeln überhaupt nicht erwähnt). Im Deutschen Kulturwart lobt Richard Groeper so etwa den "geistgeprägten Kopf", die "nordische[] Parallelität" von "Stirn-" und "Nasenlinie", die Rebel in einem seiner ausgestellten Portraits herausgearbeitet und die "schwere[n] Kampfjahre [Georges] mit dem naturalistischen Dichtergezücht" fühlbar gemacht habe. ${ }^{9}$ Sachlicher und kunstkritisch auf Rebels gestalterische Probleme bezogen, ist etwa ein Text von Fritz Nemitz in der Berliner Zeitung, der auch Rebels Bei- bzw. Unterordnung seiner Gemälde unter die ,Werkidee' und Lebensstilisierung Georges selbst in den Blick nimmt: „Das Interesse des Malers für die Person ist oft so stark, dass es der malerischen Behandlung Konkurrenz macht. Die Eigenlebendigkeit und Eigengesetzlichkeit der Farbe wird zugunsten der dekorativen Wirkung vernachlässigt. Man könnte von angewandter Farbe sprechen. Doch was an dieser Porträtreihe vor allem interessiert, ist die Art, der 'Stil', in dem die Gestalt Georges erscheint [...]. Tritt etwa bei Nietzsche, von dem George entscheidende Einflüsse erfuhr, das Private ganz hinter seinem Werk zurück, so bekommt das Subjektive, die besondere und betonte ,Haltung' bei George wesentliche Bedeutung. $\mathrm{Ja}$, es gibt kaum einen Künstler jener Zeit, bei dem Privates und Oeffentliches, bei dem individuelle Lebensform und Wirkung nach aussen so miteinander verschmolzen sind wie bei George." 10 Heute ist Carl Max Rebel weitgehend vergessen. Das Allgemeine Künstler-Lexikon: Die Bildenden Künstler aller Zeiten und Völker beispielsweise führt inn nicht. Unsicher ist zudem, wieviele Bilder des 1954 verstorbenen Malers sich erhalten haben. Denn auch wenn er in seinen Lebenserinnerungen von zahlreichen Ausstellungen und guten Verkäufen berichtet, ${ }^{11}$ gehören nach eigener Aussage mehr als 500 Gemälde aus seinem Berliner Atelier zum Kriegsverlust durch Bombenbrandschaden im November 1943.

Maik Bozza 